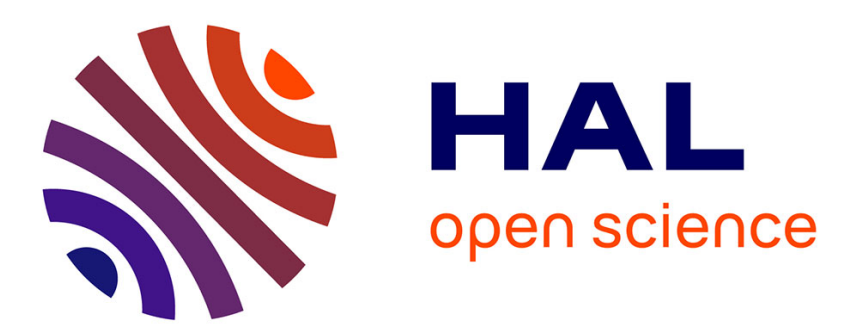

\title{
Influence of the driving waveform on the open-loop frequency response of MEMS resonators with nonlinear actuation schemes
}

Alexis Brenes, Jérôme Juillard, Laurent Bourgois, Filipe Vinci dos Santos

\section{- To cite this version:}

Alexis Brenes, Jérôme Juillard, Laurent Bourgois, Filipe Vinci dos Santos. Influence of the driving waveform on the open-loop frequency response of MEMS resonators with nonlinear actuation schemes. Journal of Microelectromechanical Systems, 2016, 25 (4), pp.812 - 820. 10.1109/JMEMS.2016.2560260 . hal-01357855

\section{HAL Id: hal-01357855}

https://hal-centralesupelec.archives-ouvertes.fr/hal-01357855

Submitted on 30 Aug 2016

HAL is a multi-disciplinary open access archive for the deposit and dissemination of scientific research documents, whether they are published or not. The documents may come from teaching and research institutions in France or abroad, or from public or private research centers.
L'archive ouverte pluridisciplinaire HAL, est destinée au dépôt et à la diffusion de documents scientifiques de niveau recherche, publiés ou non, émanant des établissements d'enseignement et de recherche français ou étrangers, des laboratoires publics ou privés. 


\title{
Influence of the driving waveform on the open-loop frequency response of MEMS resonators with nonlinear actuation schemes
}

\author{
A. Brenes, J. Juillard, L. Bourgois and F. Vinci Dos Santos
}

\begin{abstract}
This paper deals with the influence of the shape of the driving waveform on the frequency responses of MEMS resonators under nonlinear actuation. Our models show that, at large oscillation amplitudes, these responses are strongly dependent on the shape of the actuation waveform, so that the nonlinear frequency response is not the system signature but the system signature under a specific driving waveform. The case of a MEMS resonator electrostatically-driven with a sine-, pulsed- or squarewave voltage is specifically addressed. Our models and simulations, supported by experimental evidence, predict counterintuitive phenomena resulting from the distortion of the actuation waveform by the displacement-dependent electrostatic nonlinearity. Our study emphasizes that this issue should not be overlooked in order to perform quantitative MEMS characterization in the nonlinear regime.
\end{abstract}

Index Terms - Electrostatic actuation, nonlinear dynamics, open-loop frequency response, pulses

\section{INTRODUCTION}

In this paper, we analyze the influence of the actuation waveform on the response of SDOF (Single Degree of Freedom) nonlinear MEMS resonators. We illustrate our results on capacitive resonators subject to electrostatic nonlinearities and validate them experimentally on a MEMS device.

The dynamics governing MEMS devices is highly nonlinear [1]: such forces as those induced by squeezed-film damping, electrostatic actuation, Van der Waals interactions, mechanical hardening, etc. depend strongly on the displacement of the MEMS structure and are the source of very rich and complex behaviors. They give rise to unexpected phenomena such as asymmetrical frequency responses, hysteresis or parametric resonances or antiresonances [2][3][4][5][6]. Understanding them is essential to the development of MEMS-based devices such as sensors, clocks or switches which are extremely sensitive to nonlinearities. Oscillator phase noise performance is known to depend on the nonlinear characteristics of the resonator placed in the oscillating loop [7][8]. For example, through the investigation of the relationship between the nonlinear open-loop response of the resonator and MEMS oscillator phase noise, optimal operation with phase noise minimization has been achieved for specific systems [9][10].

The aforementioned phase noise studies consider a cubic nonlinear restoring force, even though the preponderant nonlinear behavior is not necessarily polynomial, as in electrostatically-actuated MEMS resonators [1][4][5]. Furthermore, such actuation nonlinearities induce waveform-dependent phenomena resulting in different largedisplacement behavior of sine-wave [10], square-wave [11][12] or pulsed-mode [13][14][15] oscillators ${ }^{1}$. In order to optimize the phase-noise performance of a non-sinusoidal MEMS oscillator, one should then determine the nonlinear open-loop frequency response of the MEMS resonator actuated with the corresponding waveform.

Recently, several works have focused on the characterization of MEMS resonators through their nonlinear (largedisplacement) frequency response [17][18][19][20]. For SDOF systems, some of these studies have even achieved nonlinear characterization via least-squares fitting procedures [19][20]. These characterization procedures must be extended to non-sinusoidal actuation cases.

\footnotetext{
A. Brenes is with THALES Avionics SAS, 25 rue Jules Védrines, 26000 Valence, France. (e-mail: alexis.brenes@centralesupelec.fr), with GeePs (Group of electrical engineering), Paris, UMR CNRS 8507, CentraleSupélec, Univ. Paris-Sud, Sorbonne Universités, UPMC Univ. Paris 06, 3,11 rue Joliot-Curie, Plateau de Moulon F-91192 Gif-sur-Yvette CEDEX and with CentraleSupélec Advanced Analog Design Chair, 3 rue Joliot-Curie, 91192 Gif-sur-Yvette, France.

J. Juillard and L. Bourgois are with GeePs (Group of electrical engineering) Paris, UMR CNRS 8507, CentraleSupélec, Univ. Paris-Sud, Sorbonne Universités, UPMC Univ. Paris 06, 3,11 rue Joliot-Curie, Plateau de Moulon F-91192 Gif-sur-Yvette CEDEX and F. Vinci Dos Santos is with CentraleSupélec Advanced Analog Design Chair, 3 rue Joliot-Curie, 91192 Gif-sur-Yvette, France.

${ }^{1}$ The last two waveforms prove to be very simple to generate in an oscillating loop [12][14], with other advantages over sinusoidal waveforms (for example, pulsed oscillators do not require feedthrough compensation schemes [16]).
} 
In this paper, we study the influence of the actuation waveform on the nonlinear frequency response of electrostatically-actuated MEMS resonators. We consider four actuation waveforms: a sine wave, positive pulses, a succession of positive and negative pulses and a square wave. Our work is based on a theoretical analysis of the nonlinear phenomena supported by experimental measurements. We show that, at large displacement oscillation amplitudes, the frequency response of the resonator is highly-dependent on the actuation waveform, so that the nonlinear frequency response is not the system signature but the system signature under a specific actuation waveform.

This paper is organized as follows: In Section II, we present the mechanical model of a parallel-plate capacitive MEMS resonator. In Section III, the mathematical background required to determine its nonlinear frequency response is developed. In Section IV, we report the simulated frequency responses and analyze our results. The validity of our main assumptions is checked in Section $\mathrm{V}$ and our simulation results are validated through experimental measurements in Section VI. Finally, conclusions and perspectives are given in Section VII.

\section{MECHANICAL MODEL}

In this section, we establish the model of a one-sided parallel-plate resonator actuated with a $2 \pi / \omega$-periodic voltage where $V_{b}$ is a constant bias voltage, and $V_{a c t}$ the actuation voltage (see Fig. 1). We describe the behavior of resonators in the absence of cubic hardening, which is valid for the device studied in Section VI. For other resonators, such as perfectly clamped-clamped beams, a cubic nonlinear term should be considered to account for midline stretching. Its impact is studied in the supplementary material.

Writing $V(t)=V_{b}(1+v(t))$, the electrical force applied on the resonator is:

$F(t)=\frac{\varepsilon S V_{b}^{2}}{2 G^{2}}\left[\frac{1+2 v(t)+v(t)^{2}}{(1-x(t))^{2}}\right]$

where $\varepsilon$ is the permittivity of the medium between the two electrodes (e.g. vacuum), $S$ the surface of the electrodes, $G$ the gap between the two electrodes and $x$ the displacement normalized with respect to $G$. The expression (1) is valid as long as the lateral dimensions of the resonator are large with respect to the gap between the electrodes. This allows us to neglect fringing fields (as in [21]). The motion of the resonator may then be described by:

$$
m \frac{d^{2} x}{d t^{2}}+c \frac{d x}{d t}+k x=\frac{\varepsilon S V_{b}^{2}}{2 G^{3}}\left[\frac{1+2 v(t)+v(t)^{2}}{(1-x(t))^{2}}\right]
$$

where $m$ is the mass of the resonator, $k$ its stiffness and $c$ a linear damping coefficient. With the notations of Table I, this leads to:

$$
\frac{d^{2} x}{d t^{2}}+\frac{\omega_{0}}{Q} \frac{d x}{d t}+\omega_{0}^{2} x=\eta \omega_{0}^{2}\left[\frac{1+2 v(t)+v(t)^{2}}{(1-x(t))^{2}}\right]
$$

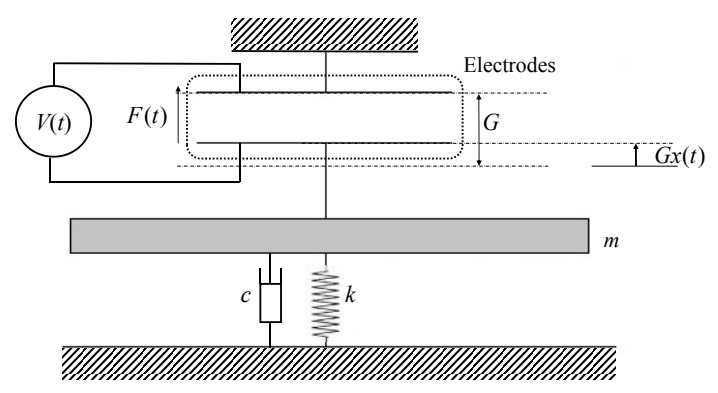

Fig. 1. Parallel-plate model of a capacitive MEMS resonator actuated by $V(t)=V_{b}+V_{a c t}(t)$. The upper electrode is fixed and the lower electrode is mobile. 
TABLE I. Table of notations.

\begin{tabular}{|c|c|c|}
\hline \multicolumn{3}{|c|}{ Actuation characteristics } \\
\hline Notation & Meaning & Formula / Order of magnitude \\
\hline$V_{a c t}(t)$ & Actuation voltage & Sine-wave : $[10 \mathrm{mV}, 1 \mathrm{~V}]$ \\
\hline$\omega$ & Angular frequency of $V_{a c t}$ & $4.2 \times 10^{5} \mathrm{rad} . \mathrm{s}^{-1}$ \\
\hline$V_{b}$ & Bias voltage & $40 \mathrm{~V}$ \\
\hline$\eta$ & Electromechanical transduction coefficient & $\frac{\varepsilon S V_{b}^{2}}{2 m \omega_{0}^{2} G^{3}}=5 \times 10^{-4}$ \\
\hline$v(t)$ & Normalized actuation voltage & $v(t)=\frac{V_{a c t}(t)}{V_{b}}$ \\
\hline$V_{0}$ & $\begin{array}{c}\text { Ratio between the first-harmonic amplitude of } V_{a c t} \\
\text { and } V_{b}\end{array}$ & ] $0 ; 0.1[$ \\
\hline \multicolumn{3}{|c|}{ Resonator characteristics } \\
\hline Notation & Meaning & Formula / Order of magnitude \\
\hline$x$ & Displacement normalized with respect to the gap & ]$-1 ; 1[$ \\
\hline$A$ & First-harmonic amplitude of the displacement $x$ & ] $0 ; 1[$ \\
\hline$\omega_{0}$ & Natural angular frequency & $\sqrt{\frac{k}{m}} \approx \omega$ \\
\hline$\varphi$ & Phase between actuation and displacement & {$[-\pi ; \pi]$} \\
\hline$Q$ & Quality factor & $\frac{\omega_{0} m}{c} \approx 10000$ \\
\hline
\end{tabular}

\section{RESOLUTION}

As mentioned in the introduction, we focus on high-Q SDOF systems. One must keep in mind that our results may not apply to complex structures exhibiting one or several modes of vibration with eigenfrequencies close to a (sub-) multiple of the frequency of interest.

Since we operate our MEMS devices with a bias voltage much smaller than the static pull-in voltage, the static component of the displacement $x$ can be neglected. Because of the large Q-factor exhibited by MEMS resonators [22][23], it is also safe to assume that $x$ can be described accurately by a single harmonic component, the higher-order harmonic content of the electrostatic force being filtered out [16]. Hence we may assume that, close to resonance, the steady-state normalized displacement of the resonator is accurately described by:

$x(t)=A \sin (\omega t+\varphi)$

where $0<A<1$. The validity of this assumption, which is sometimes referred to as the "filter hypothesis" [24], is checked in Section IV. It has also been validated experimentally in previous works [16][19].

Equation (4) may be analyzed with the method of harmonic balance, which yields two equations:

$\left\{\begin{array}{l}S(A, \varphi) \equiv \frac{1}{\omega}\left(\omega_{0}^{2}-\omega^{2}\right)-\frac{\eta \omega_{0}^{2}}{\pi A} \int_{0}^{\frac{2 \pi}{\omega}} \frac{1+2 v(t)+v(t)^{2}}{(1-A \sin (\omega t+\varphi))^{2}} \sin (\omega t+\varphi) d t=0 \\ C(A, \varphi) \equiv \frac{\eta \omega_{0}^{2}}{\pi} \int_{0}^{\frac{2 \pi}{\omega}} \frac{1+2 v(t)+v(t)^{2}}{(1-A \sin (\omega t+\varphi))^{2}} \cos (\omega t+\varphi) d t-\frac{A \omega_{0}}{Q}=0\end{array}\right.$

Equation (5) describes the frequency response of the resonator for a given actuation waveform $V_{a c t}$. The four waveforms considered in this paper are plotted in Fig. 2. The first waveform (a) is a sine-wave of amplitude $V_{s}$. The second one (b) corresponds to pulsed-mode actuation, in which positive voltage pulses of amplitude $V_{p}$ and duration $T_{p}$ are repeatedly applied at an angular frequency $\omega$. The third one (c) corresponds to "combined" pulsed-mode actuation, where positive and negative pulses of amplitude $V_{c p}$ and width $T_{p}$ are alternated at half-a-period intervals. The last one (d) is a square wave of amplitude $V_{s q}$. Note that (b) and (c) require: 
$T_{p}<\frac{2 \pi}{\omega_{0}}$.

to be considered as "pulsed-mode" actuations. In order to compare the results obtained with the four waveforms, we ensure that the amplitude $V_{1}$ of the first harmonic of the actuation voltage is the same in the four cases by setting $V_{c p}=V_{p} / 2, V_{p} T_{p}=\pi V_{s} / \omega \simeq \pi V_{s} / \omega_{0}$ and $V_{s q}=V_{s} \pi / 4$.

In the following equations, we write $V_{0}=V_{1} / V_{b}$ and assume $V_{0} \ll 1$ which is verified in our setup (see Section VI).

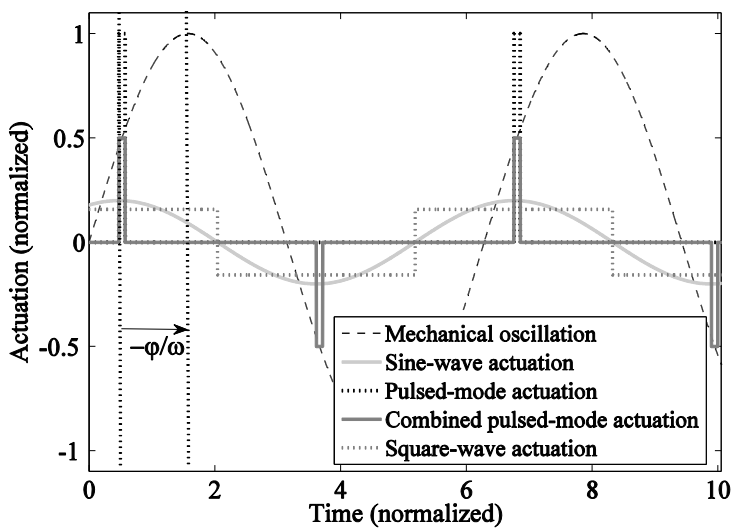

Fig. 2. Shape of actuation voltages with similar first-harmonic power $V_{0}$ : sine-wave (light-grey line), positive pulsed-mode (dark dotted line), combined pulsed-mode (dark-grey line) and square-wave (dark-grey dotted line).

From (5) we obtain:

$\left\{\begin{array}{l}S(A, \varphi)=\frac{1}{\omega}\left(\omega_{0}^{2}-\omega^{2}\right)-\frac{2 \eta \omega_{0}^{2}}{\omega\left(1-A^{2}\right)^{3 / 2}}-\frac{2 \eta \omega_{0}^{2} V_{0} F_{s}(A, \varphi)}{\omega A} \cos \varphi \\ C(A, \varphi)=-\frac{2 \eta \omega_{0}^{2} V_{0} F_{c}(A, \varphi)}{\omega} \sin \varphi-\frac{A \omega_{0}}{Q}\end{array}\right.$

where $F_{s}$ and $F_{c}$ depend on the actuation waveform. $F_{s}$ and $F_{c}$ are related to the in-phase and quadrature effects of the electrostatic force on the beam. Table II gives the expressions of these quantities for waveforms (a), (b) and (c). The expressions obtained with square-wave actuation are large and are reported in the supplementary material for the sake of readability.

The spectra of the four waveforms are very different: the spectrum of waveform (a) consists in a single peak at $\omega$, while waveform (b) contains harmonics at all the integer multiples of $\omega$, with the same power as the fundamental. The spectrum of waveform (c) contains only odd harmonics. The spectrum of (d) is a decaying spectrum made of odd harmonics. The electrostatic force is the result of the modulation of these waveforms by a displacement-dependent term, as can be seen in (1). The higher-order harmonics of the actuation waveform are spectrally folded at $\omega$, constructively or destructively, depending on their phase. This complex modulation phenomenon leads to different expressions for $F_{s}$ and $F_{c}$, as illustrated in Table II, and quite different frequency responses, as presented in Section IV. 
TABLE II

EXPRESSIONS FOR DIFFERENT ACTUATION WAVEFORMS OF SAME FIRST-HARMONIC POWER

\begin{tabular}{|c|c|c|c|c|}
\hline$V_{0}$ & \multicolumn{2}{|r|}{$\overline{V_{b}}$} & $\overline{\pi V_{b}}$ & $\pi V_{b}$ \\
\hline$F_{s}$ & $\overline{\left(1-A^{2}\right)^{3 / 2}}$ & {$\left[1+\sqrt{1-A^{2}}\right.$} & {$\left[\frac{1}{1-A \cos \varphi}\right]^{2}$} & $\left(1-A^{2} \cos ^{2} \varphi\right)^{2}$ \\
\hline$F_{c}$ & 2 & 2 & 1 & $1+A^{2} \cos ^{2} \varphi$ \\
\hline
\end{tabular}

Setting $A=0$ in the expressions of Table II yields $F_{s}=F_{c}=1$ for the four actuation waveforms, i.e. there is no impact of the waveform on the frequency response at small oscillation amplitudes. One should start accounting for these effects when $F_{s}$ or $F_{c}$ deviate significantly from 1.

Unless otherwise noted, throughout this paper, the simulated results are obtained for $Q=10000$ (typical value for standard sensors) and $\eta=5 \times 10^{-4}$.

\section{RESULTS AND DiSCUSSION}

\section{A. Qualitative analysis of the frequency responses}

The amplitude and phase responses obtained by solving (7) are represented in Fig. 3 for the four considered waveforms. For small driving voltages, the amplitude responses are identical (as shown in a previous study [16]).

Significant differences are visible wherever the oscillation amplitude exceeds $25 \%$ of the gap distance: for a given value of $V_{0}$, the maximum of the resonance curve is higher with pulsed-mode actuation than with sine-wave actuation. It is also higher with sine-wave actuation than with combined pulsed-mode actuation. As mentioned in Section III, these differences can be explained as resulting from the modulation of the actuation waveform by the displacementdependent electrostatic nonlinearity.
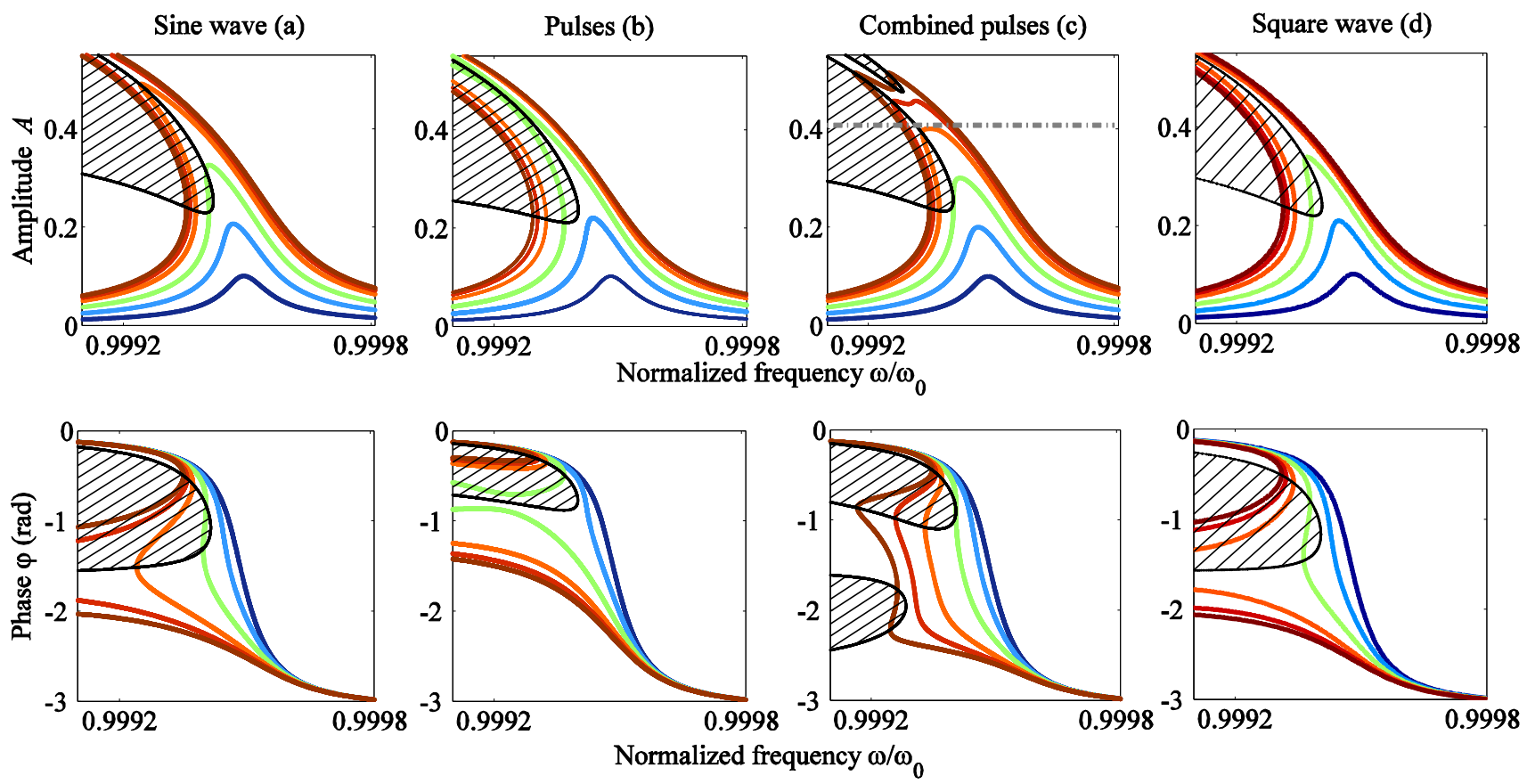

Fig. 3. Frequency responses of a plane-capacitance resonator actuated by (a) sine-wave, (b) positive pulsed-mode, (c) combined pulsed-mode and (d) square-wave actuations for increasing values of $V_{0}$ (from dark blue to brown: $V_{0}=0.01, V_{0}=0.02, V_{0}=0.03, V_{0}=0.04, V_{0}=0.045$ and $V_{0}=0.048$ ). The frequencies are normalized with respect to $\omega_{0}$. The hashed areas correspond to instability regions (see Appendix $\mathrm{C}$ in the supplementary material). The dash-dotted grey line in (c) corresponds to the amplitude above which two separate resonance peaks are found 
In the combined pulsed-mode, the amplitude response exhibits a double resonance peak above a threshold amplitude of $41 \%$ of the gap: this phenomenon is analyzed in subsection IV-B.

At large amplitudes, the responses exhibit phase and amplitude jumps, which depend on the driving waveform. For a given value of $V_{0}$, we observe that the phase jump discontinuity is larger for combined pulsed-mode actuation (c) than for the other actuation shapes (a) and (b).

Finally, the instability regions (see supplementary material for their determination) also differ from one waveform to the others. These instability regions correspond to the sets of the $(A, \omega)$ - and $(\varphi, \omega)$-spaces in which no steady-state solution of equation (6) may exist and be stable for given values of $Q$, and $\eta$. Consequently, they are the set of repelling points of the slow-time dynamics of the system, or, in other words, the set of all the unstable branches. Although their exact shapes and positions depend on $Q$ and $\eta$, it seems that their lower boundary on the phase response (corresponding to an upper bifurcation point on the amplitude response) is generally closer to quadrature for sine-wave and squarewave actuations than pulsed-mode actuations.

\section{B. Peak broadening and "double resonance" phenomenon}

An interesting and unexpected outcome of this study is the shape of the frequency response obtained with combined pulsed-mode actuation (Fig. 3-c). Above a threshold value of $V_{0}$, the frequency response exhibits a double "resonance" peak close to the stability limits. The appearance of these two maxima can be related to a "critical" amplitude, which is independent of the parameters of the system. To derive this critical amplitude, one must first notice that $C(A, \varphi)=0$ yields a relationship between the amplitude and the phase which is independent on the actuation frequency:

$R\left(A, \Gamma_{\varphi}\right)=A-2 \eta Q \omega_{0} \frac{2 V_{c p} T_{p}}{\pi V_{b}} \sqrt{1-\Gamma_{\varphi}^{2}} \frac{1+A^{2} \Gamma_{\varphi}^{2}}{\left(1-A^{2} \Gamma_{\varphi}^{2}\right)^{2}}=0$

where $\Gamma_{\varphi}=\cos \varphi$.

We may use the implicit function theorem to find the values of $\Gamma_{\varphi}$ for which $A$ is extremal, in particular:

$$
\frac{\partial A}{\partial \Gamma_{\varphi}}=0 \Leftrightarrow \frac{\partial R}{\partial \Gamma_{\varphi}}=0
$$

This yields two cases:

$$
A^{4} \Gamma_{\varphi}^{4}-A^{2}\left(2 A^{2}-6\right) \Gamma_{\varphi}^{2}+1-6 A^{2}=0 \text { or } \Gamma_{\varphi}=0
$$

Setting $\Gamma_{\varphi}=0$ in (8), this solution corresponds to:

$$
A=2 \eta Q V_{0}
$$

which may be a local maximum or minimum of the frequency response. The first polynomial has four roots:

$$
\Gamma_{\varphi}=\Gamma_{1,2}= \pm \sqrt{\frac{A^{2}-3+\sqrt{A^{4}+8}}{A^{2}}}
$$

and

$$
\Gamma_{\varphi}=\Gamma_{3,4}= \pm \sqrt{\frac{A^{2}-3-\sqrt{A^{4}+8}}{A^{2}}} .
$$

The second set of roots (13) is purely imaginary. The other set (12) is real only when $A \geq A_{l}$, where

$$
A_{l}=\frac{1}{\sqrt{6}} \text {. }
$$

If $A<A_{l}$, the amplitude response exhibits a single extremum (a maximum). If $A=A_{l}\left(\Gamma_{1}=\Gamma_{2}=0\right)$, it exhibits three coinciding extrema corresponding to a maximum of the frequency response. From (11) and (14), we find the value of the actuation voltage required to reach this threshold amplitude: 
$V_{0}=V_{0 l}=\frac{1}{2 \eta Q \sqrt{6}}$

Above this threshold (here $V_{0}=0.0408$ ), $\Gamma_{1}$ and $\Gamma_{2}$ correspond to the two maxima of the frequency response, while the extremum corresponding to $\varphi=-\pi / 2$ becomes a minimum. Note that, since $\Gamma_{1}=-\Gamma_{2}$, they correspond to values of the phase $\varphi_{1}$ and $\varphi_{2}$ that are symmetric with respect to $-\pi / 2$.

The same "double resonance" phenomenon occurs for other resonator geometries (see supplementary material). The results are qualitatively similar to the parallel-plate case, the main difference being the value of the threshold amplitude.

\section{Interpretation as autoparametric amplification}

Previous works have addressed resonance amplification and/or inhibition in parametric amplifiers [25][26], where the frequency response may sometimes exhibit several branches. As in autoparametric resonance, the phenomena we observe are related to the interaction between upper harmonics of the actuation voltage and the sine-wave displacement of the resonator.

However, the main reason why the responses observed with the two pulsed actuation cases strongly differ from the sine-wave case is because the actuation is localized within a short time-interval. This can be seen by comparing the frequency responses obtained with square-wave and sine-wave actuations. These responses are very similar, even though the spectral content of the square wave is much richer than that of a sine wave.

Some insight into the behavior of pulse-actuated resonators can be gained by noticing that the second equation of (7) can be recast as:

$$
A=-F_{c}(A, \varphi) \sin \varphi \frac{2 \eta Q \omega_{0} T_{p}}{\pi V_{b}} V_{p}
$$

whereas neglecting the electrostatic nonlinearity would yield:

$$
A=-\sin \varphi \frac{2 \eta Q \omega_{0} T_{p}}{\pi V_{b}} V_{p}
$$

We have represented in Fig. 4 the value of $F_{c}(A, \varphi)$ for sine-wave, single- and combined pulsed-mode and squarewave actuations.

In the combined pulsed-mode case, $F_{c} \geq 1$ so that $F_{c}$ may be seen as an amplification factor compared to the linear case. The "double-bump" phenomenon in Fig. 3 can then be understood as resulting from the competing effects of this amplification factor (increasing as the resonator moves away from quadrature) and the $\sin \varphi$ characteristic of the linear case.

In the case of pulsed-mode actuation, we find that $F_{c} \geq 1$ if $\varphi \geq-\pi / 2$, and $F_{c} \leq 1$ if $\varphi \leq-\pi / 2$. This explains the distortion of the phase response, and also why the maximum of the amplitude response is not at $\varphi=-\pi / 2$.

In both cases, one can also notice that $F_{c}=1$ when $\varphi=-\pi / 2$. This means that, if the resonator is used in an oscillator loop forcing $\varphi=-\pi / 2$ (when possible), the oscillation amplitude varies linearly with the amplitude of the actuation voltage.

The sine-wave case may be analyzed in the same light. Assuming $\omega \approx \omega_{0}$ in (7), the second equation becomes:

$$
A \approx-F_{c}(A) \sin \varphi \frac{2 \eta Q}{V_{b}} V_{s} .
$$

Then, $F_{c} \geq 1$ is an amplification factor independent of $\varphi$ and the frequency response looks like that of a classical "softened" Duffing resonator. Thus, in a closed-loop application at $\varphi=-\pi / 2$, sine-wave actuation is more "efficient" than combined pulsed-actuation, because of its greater amplification factor. However, this comes with the cost of a smaller resonant pull-in amplitude [14]. The same conclusion holds for square-wave actuation compared to combined pulsed-mode actuation. 


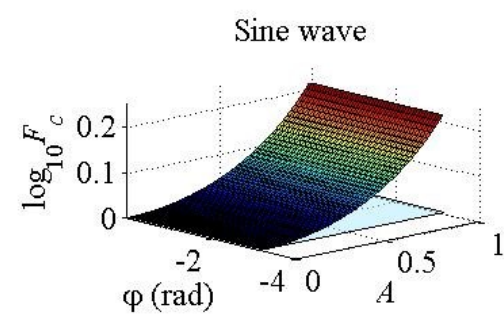

Combined pulsed-mode

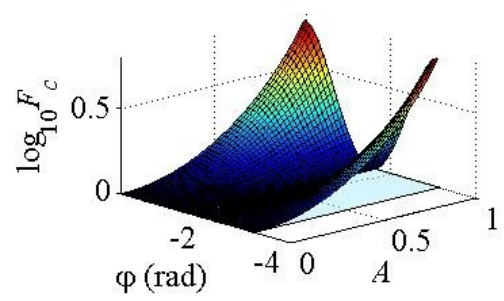

Pulsed-mode

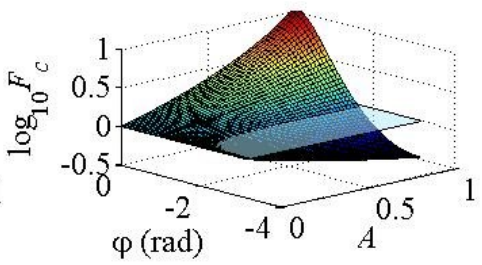

Square wave

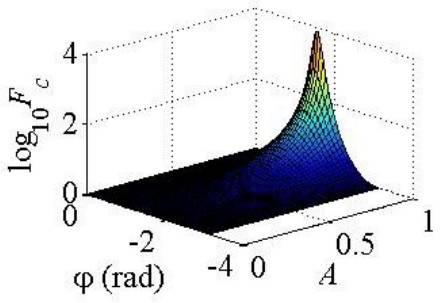

Fig. 4. Amplification factor for the four actuation waveforms. The light-blue surface corresponds to $\log _{10}\left(F_{c}(A, \varphi)\right)=0$.

\section{Comparison with Taylor-Series expansions}

In order to understand and analyze further the impact of the actuation nonlinearity, we compare the frequency responses obtained with (2) to the frequency responses obtained with Taylor-Series expansions of the electrostatic force up to the third order:

$$
\frac{d^{2} x}{d t^{2}}+\frac{\omega_{0}}{Q} \frac{d x}{d t}+\omega_{0}^{2} x=\eta \omega_{0}^{2}(1+2 v)\left(1+2 x+3 x^{2}+4 x^{3}\right)
$$

The results are reported in Fig. 5.

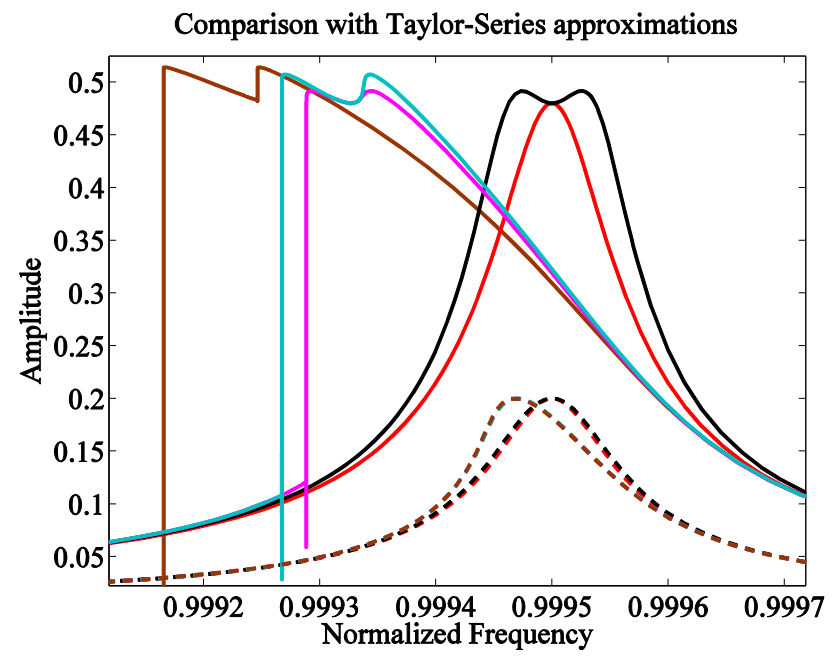

Fig 5. Transient simulations of slow frequency downsweeps with simplified models obtained from $1^{\text {st }}$-order (red curve), $2^{\text {nd }}-$ order (black curve) and $3^{\text {rd }}$-order (magenta curve) Taylor-Series expansions of the electrostatic force for $V_{0}=0.02$ (dashed lines) and $V_{0}=0.048$ (solid lines). The brown curves correspond to the model presented in the paper (no TS expansion). The brown and magenta dashed lines are almost superposed.

Fig. 5 illustrates that the modulation of the second-order term by a combined-pulsed voltage contributes to the rise of multiple resonance peaks whereas the third-order term tends to contribute to the well-known softening phenomenon. From (19) and Fig. 5, one may understand that the presence of a cubic nonlinearity in the model (e.g. due to midline stretching) would mainly result in a distortion of the amplitude response to the right (see supplementary material). 


\section{VALIDITY OF THE ASSUMPTIONS}

\section{A. Approximation of the displacement of the resonator}

To check the validity of our main assumption (4), we have performed transient simulations of (3) with MATLAB. In these simulations, the actuation frequency is swept down close to $\omega_{0}$, at a slow rate. During the sweep, we record the displacement $x(t)$ and extract its static component $A_{0}$, the amplitude $A$ and $A_{2}$ of its first and second harmonics. The results obtained for combined pulsed-mode actuation with $V_{0}=0.048$ are reported in Fig. 6 . The duty cycle of the pulsed-mode voltage in this simulation is $\alpha=T_{p} \omega_{0} / 2 \pi=1.6 \%$.

Fig. 6-a highlights a very good agreement between transient-regime simulations defined by (3) and the steady-state regime defined by (7). The presence of two resonance peaks is confirmed. On the whole frequency range, the static displacement and the second harmonic of the displacement remain inferior to $1 \%$ of its first harmonic (with the only exception of transient effects). Hence, despite the existence of high-order harmonics in the actuation voltage, the displacement of the resonator can be considered as a pure sine-wave.

(a)
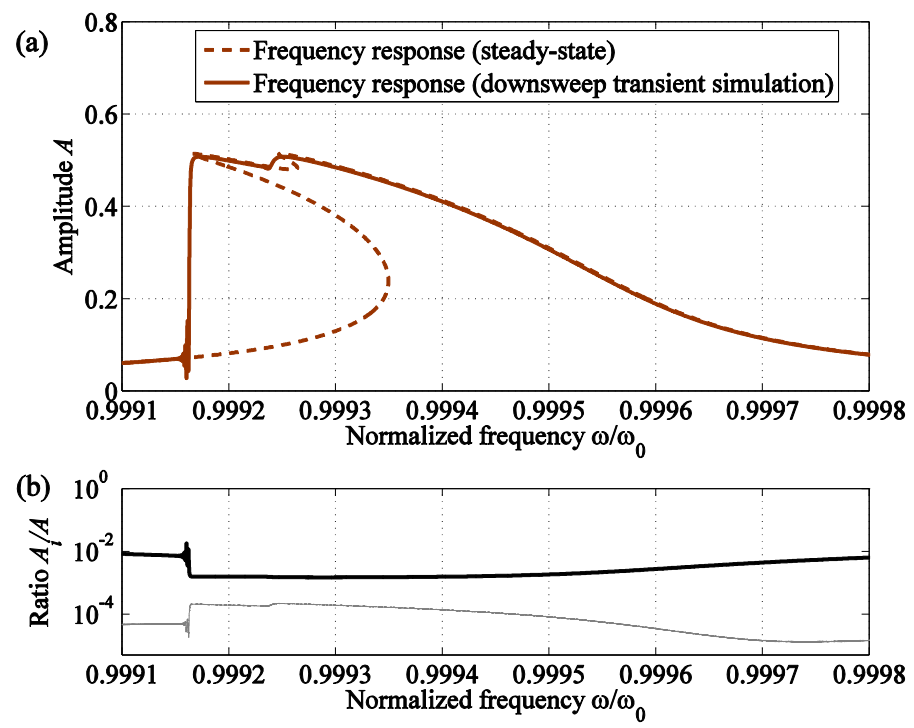

Fig. 6. (a) Comparison between transient and steady-state regime simulations for $V_{0}=0.048$. (b) Evolution of the ratios $A 0 / A$ (dark line) and $A_{2} / A$ (grey line).

\section{B. Influence of pulse width}

The phenomena observed with pulsed waveforms are strongly related to the limitation of the actuation within a very short time-interval. In order to check the influence of this pulse width, we report the results of transient simulations performed with $T_{p}=0.01 \mathrm{~s}, T_{p}=0.1 \mathrm{~s}, T_{p}=0.5 \mathrm{~s}, T_{p}=1 \mathrm{~s}$ and $T_{p}=2 \mathrm{~s}$, for $V_{0}=0.048$ in Fig. 7 . With $\omega_{0}=1$, the corresponding duty cycles are $0.16 \%, 1.6 \%, 8 \%, 16 \%$ and $32 \%$. With these parameters, the duty cycle has to be close to a few percents (e.g. $2 \%$ ) for the results to be insensitive to the width of the pulses. For larger duty cycles, the observed "double resonance" phenomenon tends to disappear.

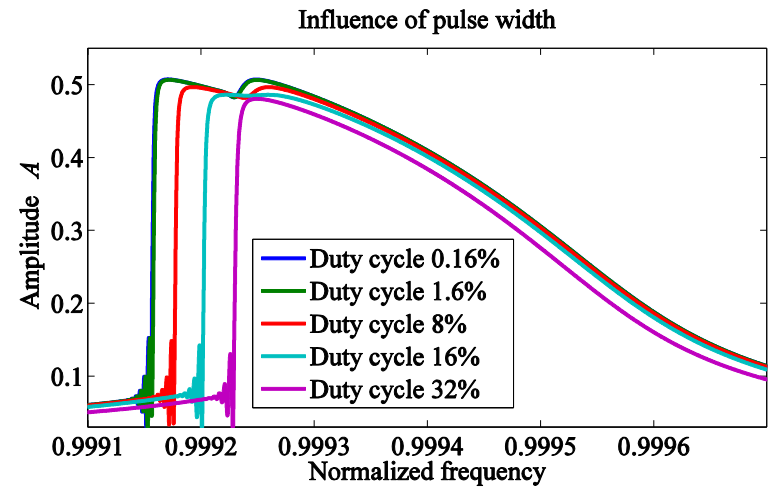

Fig. 7. Effect of pulse width on the frequency response (transient-regime simulations). 


\section{EXPERIMENTAL VALIDATION}

In this section, we report experimental results obtained with a high-Q MEMS resonator in order to validate the differences in the frequency responses predicted in Section IV, in particular the existence of a "double-resonance" associated to a phase-jump in the case of combined pulsed-mode actuation.

Since the resonator used in the experiments is an electrostatically-actuated MEMS with capacitive detection, a large parasitic capacitance is present between the input and the output of the MEMS resonant cell (i.e between actuation and detection). Hence, the frequency responses obtained with sinusoidal actuation on such a system are highly distorted [27]. They can hardly be compared either to the simulated ones or to the experimental responses obtained with the other waveforms (from which the influence of the parasitic capacitance can easily be suppressed [16]). This experimental investigation is then limited to pulsed-mode and combined pulsed-mode actuations.

\section{A. Experimental Setup}

In this section, we compare experimental results obtained with pulsed-mode and combined pulsed-mode actuation. We operate on a P90 pressure sensor from THALES Avionics made of three etched and fusion-bonded silicon wafers (see Fig. 8) [28]. It consists of a resonant rectangular beam resting on a rectangular diaphragm. One end of the beam is bonded to the diaphragm via a massive pyramidal stud, so that axial stress builds up in the beam as the diaphragm bends. Nonlinear FEM simulations show that, thanks to its imperfectly-clamped end, the resonator (i.e. the beam) is not subject to cubic nonlinear restoring forces corresponding to midline stretching. During the manufacturing process, the beam is encapsulated in vacuum to achieve a high mechanical Q-factor (between 10000 and 20000). The resonance frequency of the device is close to $65 \mathrm{kHz}$. The resonator cell is placed in an open-loop configuration shown in Fig. 9, where $C_{t}=100 \mathrm{nF}, R_{t}=1 \mathrm{k} \Omega, C_{l}=10 \mathrm{pF}$ and $R_{l}=1.0 \mathrm{M} \Omega$. In this setup, the output of the charge amplifier is the image of the motion of the resonator, possibly distorted by the one-sided capacitive detection.

In this setup, a bias voltage $V_{\mathrm{b}}=40 \mathrm{~V}$ is applied to the resonator. It is actuated by a TGA12100 waveform generator delivering pulses of width $T_{\mathrm{p}}=2 \mu \mathrm{s}$, corresponding to a duty cycle (as defined in Section V.B.) close to $13 \%$. The pulse amplitude in combined pulsed-mode actuation is set at half the amplitude of the corresponding pulsed-mode signal. We have checked that the first harmonic power (i.e. $V_{0}$ ) is equal for the two actuation waveforms. The actuation amplitudes correspond to $V_{0}=3 \times 10^{-4}, V_{0}=5.7 \times 10^{-4}, V_{0}=1.6 \times 10^{-3}, V_{0}=5.7 \times 10^{-3}$ and $V_{0}=1.1 \times 10^{-2}$.

We use subharmonic pulsed-mode actuation instead of harmonic pulsed-mode actuation since it helps overcome practical limits [16]. Here, the angular frequency $\omega$ of the input voltage pulse train is swept close to $\omega_{0} / 5$. From the output signals recorded during the frequency sweep, the amplitude and phase of the $5 \omega$-harmonic (corresponding to the motion of the resonator) are extracted after performing the nonlinear signal processing explained in a previous study [16].

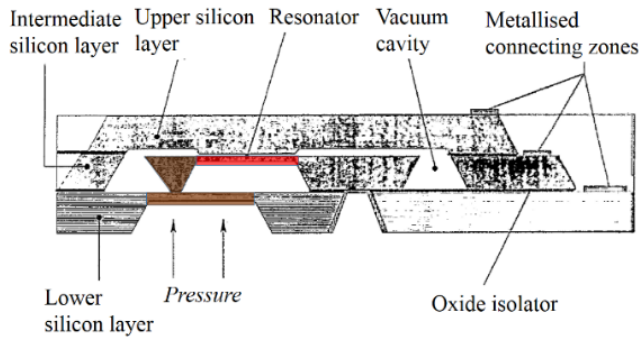

Fig. 8. Sensor structure [28]: The sensing element consists of a resonant silicon beam, with a massive stud bonded to a silicon diaphragm. The beam is actuated by the facing electrode. 


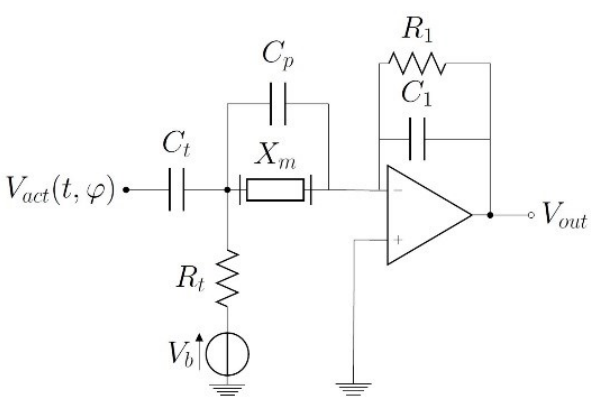

Fig. 9. Electronic architecture. The $X_{m}$-element is the resonator and $C_{p}$ an unknown parasitic capacitance.

\section{B. Limitations about the use of short voltage pulses}

When the pulses are generated at a frequency close to the natural frequency of the studied device (or "harmonic" method [16]), efficient feedthrough removal requires that the pulses be extremely short compared to the natural period of the device.

This limitation is less stringent with the "subharmonic" method [16], i.e. when the pulses are generated with a period equal to a multiple of the natural period of the device. In this case, the measured signal may still be processed and cleaned from feedthrough, even if the duration of the pulses is not so short.

In the experiments described in this paper, a relatively large duty cycle was required to provide enough power to reach the predicted behaviors (the amplitude of the actuation voltage being limited by our generator). Hence, the model presented in (7), based on pulses of infinitesimal duration, cannot be quantitatively fitted to the experimental results without adapting it to take the finite duration of the pulses into account.

\section{Experimental Results}

The measured frequency responses are reported in Fig. 10. As can be seen in Fig. 3, the difference between the frequency responses obtained with the two actuation voltages would be hard to make by sweeping the frequency up. Moreover, the "double resonance" phenomenon described in Section IV can only be observed by sweeping the frequency down. For these reasons, during each acquisition, the frequency has been swept down (slowly) and the jump discontinuities (when existing) correspond solely to upper bifurcation points. As expected, the frequency responses become more different as the actuation voltage increases whereas low amplitude results are identical.

One can notice, as assumed in Section II, the absence of mechanical hardening on the frequency responses due to the fact our resonator is not perfectly clamped at one end. As predicted, for a given actuation voltage, the peak amplitude measured with combined pulsed-mode actuation is smaller than with pulsed-mode actuation. The measured phase responses also agree well with the simulated ones. For example, the jump discontinuities on the phase responses are always larger for combined pulsed-mode actuation than for pulsed-mode actuation. At very large amplitudes $\left(V_{0}=1.1 \times 10^{-2}\right)$, the presence of the "double resonance" phenomenon on the combined pulsed-mode amplitude response is confirmed.

To ensure that this observation of the amplitude response corresponds to the predicted behavior, one can look at the phase response, which exhibits two clear jump discontinuities instead of one. Considering the displacement-to-voltage gain of the sensor and its electronics, estimated in a previous study [19] (close to 1.2V), the maxima of the frequency response of Fig. 10-b correspond to mechanical oscillation amplitudes close to $60 \%$ of the electrostatic gap $G$. At a smaller actuation amplitude $\left(V_{0}=5.7 \times 10^{-3}\right)$, the maximal mechanical oscillation is close to $40 \%$ and there is no "double resonance". The remaining differences with the model presented in Section II are related to the resonant mode of our resonator which is closer to the one of an imperfectly clamped-clamped beam (see Fig. 8) than to a parallel-plate MEMS. Taking the shape of the resonant mode into account may be performed and yields quantitatively different but qualitatively similar results. 
(a)

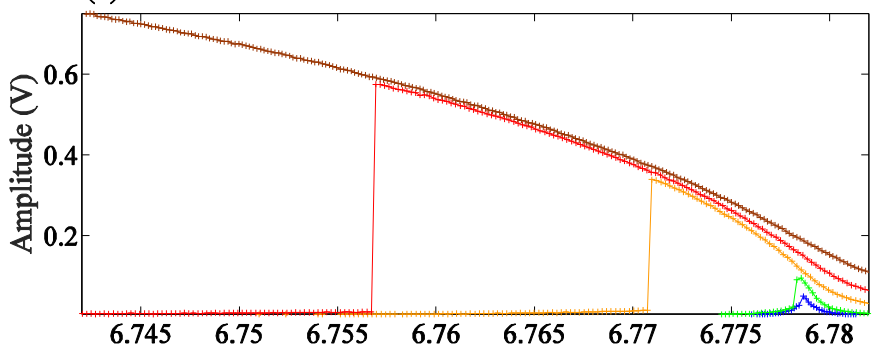

(c)

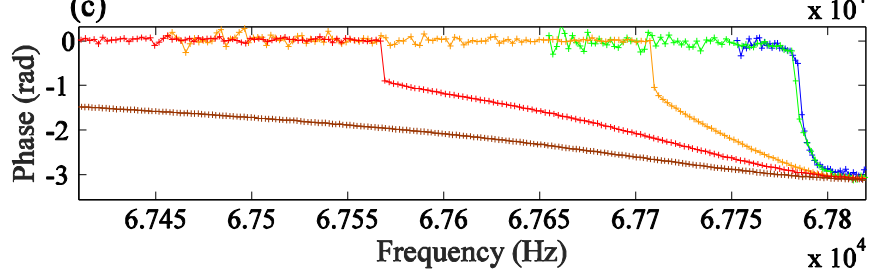

(b) Combined pulsed-mode actuation

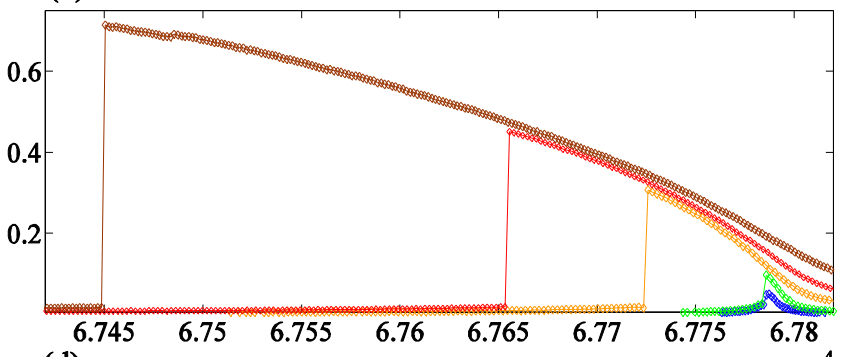

(d)

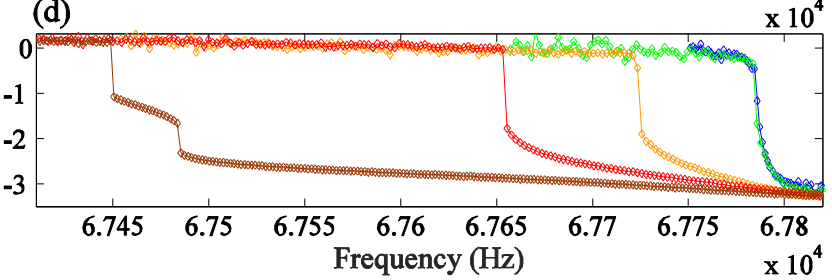

Fig. 10. Experimental sweep-down responses obtained for pulsed-mode actuation (crossed lines (a) and (c)) and combined pulsed-mode actuation (diamond lines (b) and (d)) for growing values of $V_{0}$ (from blue to brown): $V_{0}=3 \times 10^{-4}, V_{0}=5.7 \times 10^{-4}, V_{0}=1.6 \times 10^{-3}, V_{0}=5.7 \times 10^{-3}$, $V_{0}=1.1 \times 10^{-2}$.

Finally, the frequency responses obtained with pulsed-mode actuation do not exhibit this behavior, even at very large oscillation amplitudes.

\section{CONCLUSION}

In this paper, we compared the frequency responses obtained with different actuation waveforms applied on a MEMS resonator. We highlighted the dependence between the driving waveform and the nonlinear frequency response of MEMS resonators. Our conclusions are based on simulations and validated experimentally. The modulation of the actuation waveforms by the nonlinear displacement-dependent force modifies the resonance of the system dramatically, according to

the actuation scheme. This modulation can be either constructive or destructive, meaning that it can either increase or decrease the motion of the resonator compared to the hypothetical linear actuation case. All these phenomena must be taken into account in order to perform nonlinear MEMS characterization at very large amplitudes.

The presented method and results are essentially developed for MEMS under capacitive actuation. They have been illustrated on single-sided capacitive resonators but can readily be extended double-sided resonators or to other displacement-dependent actuation schemes. They can be adapted to other MEMS devices where the actuation nonlinearity is strong, i.e. whose mechanical and electrical behaviors cannot be determined independently. For instance, cantilever beams actuated by piezoelectric layers [29] may yield interesting results when actuated with nonsinusoidal waveforms. Magnetic MEMS actuated through the Lorentz force [30] may also exhibit counterintuitive nonlinear behaviors.

Knowing the dependence of the open-loop response to the actuation waveform, this study will help to determine the influence of the actuation scheme on the closed-loop phase noise of MEMS oscillators. It may also have consequences in nonlinear energy harvesting, where the actuation signal is not determined by the experimenter but by the application itself (e.g. harvesting in pacemakers). Finally, the application of the observed "double resonance" phenomenon in resonant switching devices [31] is the subject of ongoing work.

\section{REFERENCES}

[1] R. Lifshitz and M. C. Cross, "Nonlinear dynamics of nanomechanical and micromechanical resonators", Rev. of Nonlinear Dynamics and Complexity 1, pp. 1-52 (2008).

[2] S. Zaitsev, O. Shtempluck, E. Buks and O. Gottlieb, "Nonlinear damping in a micromechanical oscillator", Nonlinear Dyn. 67, pp. 860-883 (2012).

[3] C. van der Avoort, R van der Hout, J. J. M. Bontemps, P. G. Steeneken, K. Le Phan, R. H. B. Fey, J. Hulshof and J. T. M. van Beek, "Amplitude saturation of MEMS resonators explained by autoparametric resonance," J. Micromech. Microeng. 20, 105012 (2010).

[4] J. F. Rhoads, S. W. Shaw and K. L. Turner, "Nonlinear dynamics and its applications in micro- and nanoresonators: Proceedings of ASME Dynamic Systems and Control Conference (DSCC)", Ann Arbor, Michigan, USA, October 20-22, (2008). 
[5] J. F. Rhoads, S. W. Shaw and K. L. Turner, "The nonlinear response of resonant microbeam systems with purely-parametric electrostatic actuation”, J. Micromech. Microeng. 16, pp. 890-899 (2006).

[6] V. Kaajakari, T. Mattila, A. Oja, and H. Seppä, "Nonlinear limits for single-crystal silicon microresonators", J. Microelectromechanical Systems 13, pp. 715-724 (2004).

[7] B. Yurke, D. S. Greywall, A. N. Pargellis, and P. A. Busch, "Theory of amplifier-noise evasion in an oscillator employing a nonlinear resonator", Phys. Rev. A 51, 5, pp.4211-4229 (1995).

[8] D. K. Agrawal and A. A. Seshia, "An analytical formulation for phase noise in MEMS oscillators", IEEE Transactions on Ultrasonics, Ferroelectrics and Frequency control, 61, 12, pp. 1938-1952 (2014).

[9] E. Kenig, M. C. Cross, L. G. Villanueva, R. B. Karabalin, M. H. Matheny, R. Lifshitz, and M. L. Roukes, "Optimal operating points of oscillators using nonlinear resonators", Phys. Rev. E 86, 056207, pp.129-132 (2012).

[10] L. G. Villanueva, E. Kenig, R. B. Karabalin, M. H. Matheny, Ron Lifshitz, M. C. Cross, and M. L. Roukes, "Surpassing fundamental limits of oscillators using nonlinear resonators", Phys. Rev. Letters 110, 177208 (2013).

[11] A. Fargas-Marques, J. Casals-Terré, and A. M. Shkel, "Resonant pull-in condition in parallel-plate electrostatic actuators", J. of Microelectromechanical systems, 16, 5, pp. 1044-1053 (2007).

[12]J. E.-Y. Lee, B. Bahreyni, Y. Zhu, and A. A. Seshia, "A single-crystal-silicon bulk-acoustic-mode microresonator oscillator", IEEE Electron Device Letters, 29, 7, pp. 701-703 (2008).

[13] S. Gorreta, D. Fernandez, E. Blokhina, J. Pons-Nin et al., "Pulsed digital oscillators for electrostatic MEMS", IEEE Transactions on Circuits and Systems 59, pp. 2835-2845 (2012).

[14]J. Juillard, A. Bonnoit, E. Avignon, S. Hentz and E. Colinet, "Large amplitude dynamics of micro/nanomechanical resonators actuated with electrostatic pulses", J. Appl. Phys. 107 , 014907 (2010).

[15]A. Brenes, J. Juillard and F. Vinci Dos Santos, "Electrostatically-induced modal crosstalk phenomena in resonant MEMS sensors: Proceedings of the 16th Symposium on Design, Test, Integration \& Packaging of MEMS and MOEMS”, Cannes, France, 1-4 April, pp. 294297 (2014).

[16]A. Brenes, J. Juillard, F. Vinci Dos Santos and A. Bonnoit, "Characterization of MEMS resonators via feedthrough de-embedding of harmonic and subharmonic pulsed-mode response", Sens. Actuators A 229, pp. 211-217 (2015).

[17]W. O. Davis, "Measuring quality factor from a nonlinear frequency response with jump discontinuities", J. of Microelectromechanical systems, 20, pp. 968-975 (2011).

[18]J. M. Londoño, S. A. Neild and J. E. Cooper, "Identification of backbone curves of nonlinear systems from resonance decay responses", J. Sound and Vibration 348, pp. 224-238 (2015).

[19]A. Brenes, J. Juillard, L. Bourgois and F. Vinci dos Santos, Parameter estimation from nonlinear frequency response of MEMS resonators: Proceedings of the 2015 International Symposium on Circuits And Systems (ISCAS)", Lisbon, Portugal, 24-27 May 2015, pp. 1318-1321 (2015).

[20]A. J. Dick, B. Balachandran, D. L. DeVoe and C. D. Mote Jr, "Parametric identification of piezoelectric microscale resonators", J. Micromech. Microeng. 16, pp. 1593 - 1601 (2006).

[21]M. I. Younis, F. M. Abdel-Rahman and A. Nayfeh, "A reduced-order model for electrically actuated microbeam-based MEMS", J. of Microelectromechanical systems, 12, 5, pp. 672-680 (2003).

[22]J. E.-Y. Lee and A. A. Seshia, "Square wine glass mode resonator with quality factor of 4 million: Procedings of the $7^{\text {th }}$ IEEE SENSORS Conference", Lecce, Italy, 26-29 October 2008, pp. 1257-1260 (2008).

[23]S. Ghaffari, S. A. Chandorkar, S. Wang, E. J. Ng, C. H. Ahn, V. Hong, Y. Yang and T. W. Kenny, "Quantum limit of quality factor in silicon micro and nano-mechanical resonators", Scientific Reports 3, 3244 (2013).

[24]A. Gelb, W. E. Vander Velde, "Multiple-input describing functions and nonlinear system design", McGraw-Hill, Chapter 3, 1968.

[25]J. F. Rhoads and S. W. Shaw, "The impact of nonlinearity on degenerate parametric amplifiers”, Applied Physics Letters 96 (23), 234101 (2010).

[26]J. F. Rhoads, S. W. Shaw, K. L. Turner, J. Moehlis, B. E. DeMartini and W. Zhang, "Generalized parametric resonance in electrostatically actuated microelectromechanical oscillators", J. of Sound and Vibration 296, pp. 797-829 (2006).

[27]G. Vidal-Alvarez, F. Torres, N. Barniol and O. Gottlieb, "The influence of the parasitic current on the nonlinear electrical response of capacitively sensed cantilever resonators", J. Appl. Phys. 117, 154502 (2015).

[28]J. Mandle, O. Lefort and A. Migeon, “A new micromachined silicon high accuracy pressure sensor", Sens. Actuators A 46-47, pp.129-132 (1995).

[29]K. Wolf and O. Gottlieb, Technical Report No. ETR-2001-02, "Nonlinear dynamics of a cantilever beam actuated by piezoelectric layers in symmetric and assymetric configuration", Technion Israel Institute of Technology (2001).

[30]John A. Pelesko and David H. Bernstein, Modeling MEMS and NEMS (Chapman \& Hall/CRC, 2003), p. $273-278$.

[31]Y. Lin, W.-C. Li, Z. Ren, and C. T.-C. Nguyen, "A resonance dynamical approach to faster, more reliable micromechanical switches: Proceedings of the 2008 IEEE International Frequency Control Symposium”, Honolulu, HI, 19-21 May, pp. 640-645 (2008).

[32]J. Juillard, G. Arndt and E. Colinet, "Modeling of micromachined beams subject to nonlinear restoring or damping forces", J. of Microelectromech. Syst. 20, pp. 165-177 (2011).

[33]J. Juillard, “Analysis of resonant pull-in of micro-electromechanical oscillators”, J. Sound and Vibration 350, pp. 123-139 (2015). 